\title{
Videogame Walkthroughs in Educational Settings: Challenges, Successes, and Suggestions for Future Use
}

\author{
Kelly Bergstrom \\ University of Hawai'i at \\ Mānoa \\ kelly.bergstrom@hawaii.edu
}

\author{
Emily Flynn-Jones \\ York University \\ emilyfj@yorku.ca
}

\author{
Jennifer Jenson \\ York University \\ jjenson@edu.yorku.ca
}

\author{
Cristyne Hebert \\ York University \\ cristyne_hebert@edu.yorku.ca
}

\begin{abstract}
In this paper we describe the use of videogame walkthroughs implemented in three different educational contexts: 1) an informal learning environment where 11-12 year-olds used text and video walkthroughs to supplement their Legend of Zelda: Wind Waker play during an optional, lunch-time video game club in their school library; 2) a formal learning environment where grade six (11-12 year-old) students used a written walkthrough for Lost Winds 2: Winter of the Melodias played as part of their Language Arts classroom; and 3) the use of a written walkthroughs by Grade 7 and 8 teachers as part of their preparations to teach a game-based learning unit in their Geography classrooms. Taken together, we argue that while walkthroughs can be useful pedagogical tools when using videogames in classroom settings, the social and cultural contexts in which they are introduced must be carefully considered in order for their effectiveness to be fully realized.
\end{abstract}

\section{Introduction}

In order to address the unique skills and needs of a new generation of students, it has been argued that classroom practice must specifically accommodate the 'digital affinity' of the so-called 'net generation' who are connected, creating, and likely know more new technological developments than their teachers [16, 17]. The belief that youth exhibit a digital affinity undergirds the push towards an inclusion of digital games in the classroom. Supplementing a curriculum with games builds on the out of school activities of these digitally connected youth, as many are already playing games, especially boys and young men, in their leisure time [14, 27, 33]. Furthermore, it is argued that - at least for some students - games may be more engaging than the books, worksheets, or lectures that have, for some time, been commonly used as teaching tools $[26,29,30]$.
This paper takes up those calls for games to be, much like radio, television and cinema, learning environments that can be studied, explored and experienced. We ask: how might both teachers and students who are unevenly skilled when it comes to playing digital games best be supported to use them in formal learning environments? Relatedly, how have videogame 'walkthroughs' been used as tools to support learning as well as videogame skill development? And finally, what are some of the potential areas of concern that must be acknowledged in order for walkthroughs to be an effective learning aid in the classroom? As games have made their way into a variety of in/formal learning contexts with both negative [31] and positive impacts [34] on the learning outcomes, it is increasingly important to study how to best support novice players and pedagogues. And, as reporting on this research remains heavily focused on gameplay and/or student interactions with the technology, there needs to be increasing attention paid to peripheral materials and cultural contexts that also constitute key components of the gameplay experience.

Consalvo $[11,12]$ has argued for the inclusion of peripheral materials, or paratexts, in the study of digital games and their surrounding communities, as such sources "serve a specific role in gaming culture and for gaming capital; they instruct a player in how to play, what to play, and what is cool (and not) in the game world" (p. 22). Beyond developer-created texts (e.g. instruction manuals), a vast array of playercreated knowledge also exists about particular games including art [8]; calculations on the underlying mathematical mechanics of the game [21, 24]; thirdparty modifications ('mods') and 'add-ons' to the game client [10, 32]; and MMOGs and other games more generally, as demonstrated through conversations on message boards and forums $[5,6,24]$.

Videogame 'walkthroughs' are a form of paratexts produced by players. They are most easily defined as texts that describe where to go in the game, how to solve puzzles, and how to successfully progress to the end. Building on Consalvo's arguments that texts outside of a game not only matter, but are integral to 
understanding play, the goal of this paper is to add to the growing discussions about games and education by means of reporting on the uptake and use of paratexts in both formal and informal learning environments.

\subsection{Motivations and Scope}

The specific type of paratext at the centre of this study is the videogame walkthrough. These are written or video documents created by players about a game, with an intended audience of other players seeking assistance through a step-by-step guide explaining how to complete in-game objectives and tasks. Typically, walkthroughs are used to supplement digital gameplay in a leisure context. Walkthroughs can also be useful for supporting and scaffolding digital gameplay in educational contexts, especially for students and teachers who are new to videogames and their affordances. In this paper we demonstrate that as valuable walkthroughs can be, their use value may not be readily apparent to novice players. Drawing from our own fieldwork in a variety of contexts, we provide examples of the variance in reception, largely a result of how walkthroughs were framed when introduced to our research participants. To this end, we describe the uptake (or lack thereof) of videogame walkthroughs implemented in three different contexts:

1) An informal learning environment where 1112 year-olds used The Legend of Zelda: The Wind Waker text and video walkthroughs during an optional, lunch-time videogame club in their school library;

2) A formal learning environment where grade six (11-12 year-old) students used a written walkthrough for Lost Winds 2: Winter of the Melodias played as part of their Language Arts classroom;

3) The use of a written walkthroughs by Grade 7 and 8 teachers as part of their preparations to teach a game-based learning unit in their Physical Geography classrooms.

In this paper, we are less concerned with the evaluation of whether adding digital gameplay to an educational environment resulted in measurable learning gains than sharing lessons learned from our observations of the use of walkthroughs in varying educational settings. In so doing, we aim to highlight how audiences interact with games and paratexts is highly dependent on social and cultural contexts. What we hope to illustrate is that digital gameplay is a socially mediated experience even in an explicitly educational environment. Related to this point is that how walkthroughs are framed can have unintended consequences that limit uptake by students. Additionally, we illustrate that walkthroughs can act as bridging texts for teachers who are otherwise unfamiliar with videogames, but seek to integrate digital games in their classroom or use them to supplement existing curriculum. We conclude this paper with a discussion of the ways videogame walkthroughs can be used as a means of reinforcing media and/or information literacy, offering suggestions for how they can be successfully introduced to in/formal learning environments.

\section{Videogame Walkthroughs in Educational Contexts}

In his dissertation on the topic, Giddings defines walkthroughs as such: "[A walkthroughs is] in part an instruction manual, assisting a stuck videogame player through a particular puzzle, tortuous labyrinth or fiendish demand on hand-eye co-ordination, but it is also a verbal map of the peculiar space-time of the videogame's virtual world. It is a document of the writer/player's skill and effort in exploring and solving every last aspect of the gameworld. It is knowledge shared via the Web to both assist other players and to display the writer's expertise in, and devotion to, the game in question. A walkthrough is a determinedly non-literary work, dispassionate, stripped of noninstrumental description and of most subjective reflection on its writer's part. The walkthrough's audience is addressed in an impersonal second person - the player instructed" [15:31].

Videogame walkthroughs are a form of paratext that are typically produced by fans or enthusiasts of the game, rather than officially put out by the game's developers. Juul [18] makes a distinction between two types of supplemental guides, walkthroughs and strategy guides, based on the type of game being played: "Progression games have walkthroughs: lists of actions to perform to complete the game. Emergence games have strategy guides: rules of thumb, general tricks" (p. 328). As walkthroughs provide step-by-step details about the tasks that must be completed to finish a game, they may contain spoilers by providing information about crucial plot points in a game's narrative. In this paper we focus our attention on what are considered walkthroughs according to Juul's definition, that is, texts that allow players to follow along step by step to complete the objectives laid out for a player to successfully complete the game.

As mentioned above, Consalvo [11, 12] has argued that accounting for paratexts (such as walkthroughs) is an essential part of understanding videogames in a broader cultural context. Apperley and Beavis [1] extend Consalvo's argument, describing how students used and also made their own paratexts as part of the 
game-based pedagogy in their high school classrooms where they argue that there are multiple forms of information literacy at work. First, students must find a walkthrough (practicing research skills) and then evaluate its reliability (practicing critical evaluation skills). They must then take the information they consumed in one form (e.g. written text or video) and then apply it to another context (e.g. making their way through a maze in a game) (p. 135). Walsh and Apperley [35] have also published research surrounding paratexts in the high school classroom, arguing that the integration of paratexts is necessary for students' further refinement of their gaming literacies.

Other successful integrations of walkthroughs into a classroom include Stanley and Mawer [28] who discuss walkthroughs as part of their larger argument for the inclusion of digital games in the English as a Second Language classroom. Taking the same rationale as Gee and others [14, 26], Stanley and Mawer argue that games provide an enjoyable means to practice one's language skills. Not only do walkthroughs offer step-by-step instructions about how to complete a game to allow students to troubleshoot when they run into difficulties, but they also can be used as part of pair exercises for practicing dictation and descriptive language, where one student must read and relay instructions to another student who is trying to play the game (pp. 4-5).

Vie [34] also provides an example of how walkthroughs can be mobilized in the classroom, in this case describing how writing walkthroughs serve as an exercise for college students to practice technical writing skills that might be more interesting than traditional technical writing assignments such as resumes or usability studies (p. 158). A similar project is assigned by Burn [7], who after having students design a game, has them write a walkthrough to reinforce peripheral literacies.

Kringiel [19] argues that walkthroughs make up an important part lesson planning when teaching students about media literacy using videogames. Citing the learning objective of "media use," which he defines as "(a) the competence needed to make receptive use of the medium as well as (b) the competence necessary for interactive usage of the medium” (p. 636), Kringiel suggests, much like the observations relayed by Apperley and Beavis, a student can demonstrate competency by finding and then using walkthroughs in a successful manner (p. 637).

Throughout the literature reviewed thus far, walkthroughs were successfully added to a classroom with minimal resistance on the part of the students. We agree that walkthroughs offer up opportunities to reinforce media and information literacy goals in both formal and informal learning environments. Before moving on to a discussion of these possibilities, we describe three examples from our fieldwork where we highlight both the successes and challenges of using walkthroughs in differing educational contexts. We do so as examples of the diverse ways walkthroughs can be used to scaffold specific learning objectives, but also to highlight potential pitfalls and areas of concern, especially for researchers and/or educators working with students who may not have extensive videogame play experience or who may not have previously used walkthroughs in their leisure time.

\section{Observations from the Field}

The observations presented in this paper come from fieldwork conducted as part of two larger research projects. The studies involving The Legend of Zelda: The Wind Waker and Lost Winds 2: Winter of the Melodias were part of a multi-year SSHRC funded project. The study that included Sprite's Quest, an educational game developed by the Ontario Ministry of Education to supplement the existing Physical Geography curriculum, was part of a separate year long provincially funded project.

In our review of other studies investigating the use of digital games as part of a language arts or geography curriculum, we observed few examples of researchers providing a detailed summary of how a game was integrated into the day-to-day workings of an elementary or middle school classroom. As with our publication efforts across this and other projects (see for example $[2,3,9]$ ) we describe in detail our study design and method for collecting data. It is hoped that this information will be of use for researchers investigating similar questions to our own and/or aid in replication efforts in new contexts and study environments.

\subsection{Study 1: Adding Walkthroughs to a Lunchtime Gaming Club}

In this first example, students were invited to participate in an optional lunchtime club. This club was part of a pilot study conducted to determine the level of intervention required if digital games were to be used to supplement the existing Grade 6 (students aged 1112) Language Arts curriculum. Here, students were invited to play The Legend of Zelda: The Wind Waker (herein shortened to Wind Waker) on the WiiU. Club meetings were held once a week over the lunch hour in the partner school's library. Rather than using a randomized sample, participants were recruited by our partner teacher who was familiar with the schedules 
and extracurricular commitments of the Grade 6 students.

Three separate iterations of the club ran over the course of the school year. The first two clubs were mixed sex groups comprised of students who had not previously played Wind Waker. The first club had 12 participants (six girls and six boys) and the second club had 13 participants (six girls and seven boys). The third club was girls only and had nine of the previous participants return, tasked with acting as peer mentors to assist nine new participants to learn how to play this game. A detailed discussion the study design and the results of this pilot study are detailed in a previous publication [9].

Wind Waker had been selected because the Zelda franchise has a reputation for producing narratively rich games. However, Wind Waker's frequent jumping puzzles and side quests to find specific items served as roadblocks for the participants not as familiar with the tropes and hidden expectations that would be known to those who had previous experience with the roleplaying game genre. Because these early puzzles acted as roadblocks, participants did not progress to the later portions of the game where more of Wind Waker's story would be revealed. In some cases, participants were unable to discern the difference between actual side-quests, and the distractions that the game's designers added as 'Easter Eggs'. We also observed that our more novice participants were not as technically skilled and found it difficult to press the WiiU controller's multiple buttons correctly and quickly enough to successfully complete jumping puzzles or fighting tutorials. Rather than having a member of the research team complete the puzzles on the students' behalf, text-based walkthroughs were introduced to the club. The goal was to allow students to problem solve their difficulties, by providing access to step-by-step directions about how to complete the troublesome puzzles.

Hoping to model the information seeking and evaluation behaviours described by Apperley and Beavis [1], we sought out and selected a Wind Waker walkthrough that was easily found via Google and contained all the required information needed to complete this game. We selected a text-based walkthrough because the specific steps required to successfully complete particular tasks were laid out in plain English, written at a level appropriate for Grade 6 students, and did not include any inappropriate language [36]. For example, we observed that many of the novice participants were having difficulty in the fighting tutorial, which must be completed in order to unlock a sword and continue your progression in the game. The walkthrough we selected outlines the specific steps required to win a fight against Orca, the trainer who will eventually reward you with a sword. These steps were as follows:

1. "Use B (without touching D-stick or L) to hit him. Repeat.

2. Hold down L, and use B (without touching Dstick) to hit him. Repeat.

3. Hold down L, press the D-stick up, and use B to hit him. Repeat.

4. Stand back from Orca, press and hold B until Link has his sword behind him, then walk up to Orca and release B. Repeat.

5. This is the important one: hold $\mathrm{L}$ and wait for your controller to vibrate. You will hear a noise, your sword will turn green, and the A button in the top right corner will light up. Press A. Repeat.

6. Hold L and hit A (don't wait for controller to vibrate) to hit him. Repeat.

Now that you have a sword, you can go around and cut grass to find rupees." [36].

The walkthrough was available on a PC located in same the room as the WiiU play stations. The walkthrough proved unpopular for two reasons: first, the supervising teacher introduced the resource a 'cheat', which immediately stigmatized the use of the walkthrough. Second, students were reticent to consult the walkthrough even when they were stuck because they would have to walk across the library making the rest of the group aware that they were 'cheating'. According to research field notes, none of the participants in the first club consulted the walkthrough.

For the second club, researchers introduced the walkthroughs to participants in their first play session. Each pair was given an iPad connected to a wireless Internet connection via a portable router provided by the research team. The text-based walkthrough described above was loaded in the browser for students to use. The immediate access to walkthroughs was introduced to prevent groups from falling behind and as a resource that could help students solve problems themselves if they were stuck, and the use of iPads was intended to allow access without having to walk across the library, as was the case in the first club. Researchers recommended to the group that each pair split up the workload so that while one partner was playing, the other could use the walkthrough and act as a 'navigator' so that they were both had an active role at all times.

The majority of participants used the walkthroughs as intended, but the addition of the iPads to the club had some unexpected consequences. We did not anticipate that some of the male pairs would eschew text walkthroughs in favour of video-based aids with player audio commentary on YouTube. This was likely 
because it is easier to follow a video. Participants could match game locations and copy what they saw on screen with less effort than it was to read and interpret text to work out where they were in the game and what they needed to do. While industrious, no content filters had been applied to the iPads and some of the content located by students featured graphic, age inappropriate language. The introduction of the iPads had another unintended consequence; the device facilitated discreet disengagement. One participant, for instance, asked a researcher if they could use it to look at Facebook (a site with a minimum age requirement of 13 , at least a year older than a sixth-grader). Despite being told not to, they accessed Facebook anyway and proceeded to spend the rest of the session browsing the site while their partner played alone and without the aid of the walkthrough.

Participants from the first two clubs were invited to return to serve as mentors for the third club. Each team had at least one experienced or 'expert' player, and we observed the walkthroughs were utilized less than in previous clubs. We hypothesize, based on existing research [13], the confidence of previously novice players increased and they trained newcomers in the use of the walkthrough as well as how to play the game. Many of the returning players remarked on their improved proficiency with the game and their confidence in the role of expert within their playgroups, reducing the need to consult the walkthroughs on the iPads.

\subsection{Study 2: Using Walkthroughs to Address Language Arts Curriculum Objectives}

The second example comes from a two-week classroom intervention where 60 students ( 32 boys and 28 girls) played a game as part of their Grade 6 (students aged 11-12) Language Arts curriculum. The goal of this study was to add more empirical evidence to support the claim that games can act as a learning tool in formal educational environments. Specifically, the students played Lost Winds 2: Winter of the Melodias (herein shortened to Lost Winds 2), a commercial puzzle and adventure game available on the iTunes Store. This particular game was selected because it is age appropriate (rated E), and because of the large amount of reading required to complete gameplay. Further, it was chosen because it was available through the iTunes store, meaning it could be played on iPads and therefore could be played by larger number of students and was more portable than a console requiring a TV and controllers.

Prior to the launch of this intervention, researchers and teachers worked together in a full day collaborative curriculum writing retreat. Daily lesson plans, homework, assignments, and assessment tools were created at this time. At the end of the retreat the teachers were each loaned an iPad to be used for the remainder of the study period, but also so they could play Lost Winds 2 in advance of their students. When we checked in with the teachers closer to the start date of the classroom intervention, we found that the teachers all had difficulty progressing through the game due to unfamiliarity with the touchscreen mechanics of a game played on an iPad. The teacher who did finish the game noted that her daughter played on her behalf. This observation is important, as it means that none of the teachers in the study were personally familiar with the overall story arc of Lost Winds 2. To remedy this, researchers provided an answer key for the daily questions to assist teachers with their grading. We will return to this issue in the third example, were we outline how walkthroughs were used as a workaround for teachers who lacked the technical skills needed to complete the videogame at the centre of the curriculum.

On the first day of the classroom intervention, our partner teachers introduced the project to the students. After the research team introduced themselves to the students and led a discussion of the overall goals of the project, the teacher led a short lesson and prompted students to reflect upon the components of a narrative. Finally, students were asked to complete an intake survey in order for researchers to better understand their attitudes towards videogames as well as their prior play experience. Alongside this intake survey, students completed a pre-assessment based on Ontario's standardized Grade 6 literacy assessment.

From day two to the end of the study, students played in randomly assigned single-sex pairs. For the duration of the study, each pair was assigned their own iPad mini that researchers had pre-loaded with Lost Winds 2 and a bound, printed copy of a detailed fancreated written walkthrough text for the game. Using the same criteria as in Wind Waker example, the walkthrough was selected because it was clearly written in plain English and provided a complete (and detailed) overview of every step required to complete the game [20]. Each individual student was also given a workbook where they were asked to keep their assigned work for this unit. This work included daily questions to assess how well the students had read and understood the narrative of the game, and small assignments that were completed every other day that were designed to have students make connections between the game and the larger learning objectives of the unit. 


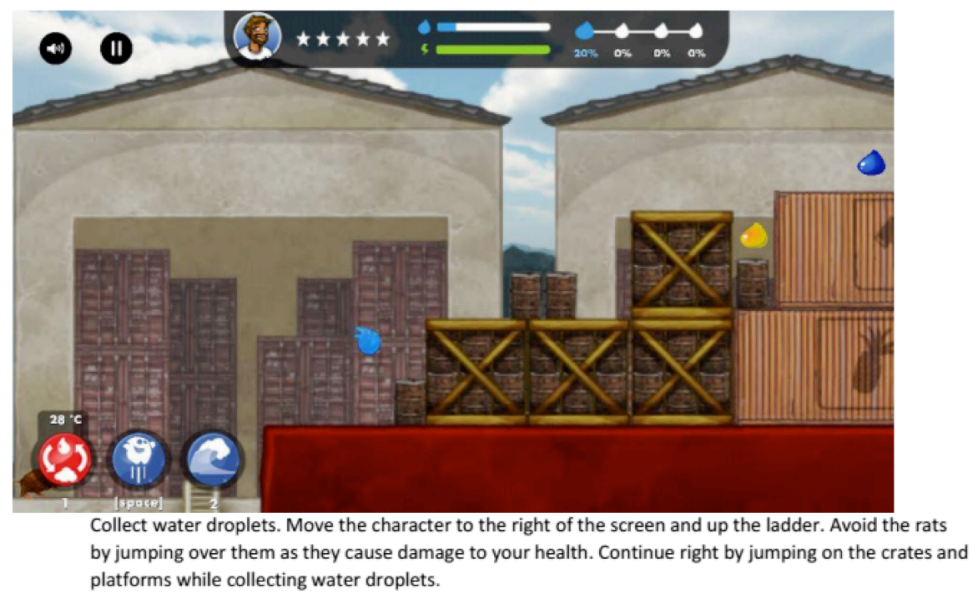

Figure 1: Screen capture of the walkthrough created for Sprite's Quest. Pictured here is a screenshot of what the player would see on their own screen. The text below provides step-by-step instructions about what actions the player should take to continue their progression in the game.

While the walkthroughs were presented as an essential reference text to be used as part of the Lost Winds 2 curriculum, uptake by the students was inconsistent. Some students sat side by side and one student would work the controls and the other one would literally read from the walkthrough, telling the person playing where to go and what to do as they also watched over the shoulder of the person playing. Other students referred to the walkthroughs only when they were unable to progress in the game (e.g. locating a missing item required to progress to the next stage of the narrative). Sometimes students would ask researchers for help, most frequently citing "being stuck" and that they were unsure about what to do next in the game. Researchers would ask students if they had consulted the walkthrough when they were unable to advance, reminding the students of the resource sitting beside them.

Returning to the daily questions, these were meant to probe to what degree the students had understood the story that was revealed during the day's play session. The questions increased in difficulty, typically beginning by asking about particular facts from the story, then moving on to questions that required students to extrapolate or state an opinion based on the information they uncovered thus far. For example, the three question prompts from Day 1 were as follows:

1. Who were the characters in the beginning of the game?

2. From your list, what is the name of the main character that you will play throughout the game? What is something interesting that you remember about the main character?

3. What is the main character's goal at the beginning of the game?

The answers to many of these questions, especially those probing about facts, were contained in the text of the walkthrough. And yet, researcher field notes indicated that students were reluctant to open the text back up to re-read the walkthrough to find their answers. Instead, partners would argue about what they would write down, or in some cases, turn around and ask a nearby group for the answer.

\subsection{Study 3: Walkthroughs as a Teacher Aid}

For the third study, members of the research team wrote and distributed a walkthrough for an educational physical and human geography game, Sprite's Quest (Fig. 1). This walkthrough was written in the step-bystep format of the walkthroughs used in the first two studies. Building on our findings from Study 2, that teachers may not have the technical skills required to successfully complete a play through of the game prior to introducing it in their classroom, the rationale for producing the walkthrough was to enable teachers, and students for that matter, to have a guide for advancing in the game, much like enthusiast players who produce them for commercial off the shelf games. The walkthrough was first introduced to teachers in a twoday curriculum planning workshop, where we invited them to play the game, and create a game-based learning unit based on the game and its physical and human geography concepts. The walkthroughs were posted to the study's website to be accessed by teachers at any time. Of the 34 teachers who participated in the study, 5 provided copies of the walkthroughs to their classes as student learning aids. Briefly in this section, we focus on two different uses of the walkthrough, one by a teacher who was tentative about her own gameplay abilities and the other, by a student who was also anxious about her gameplay abilities.

In the first use example, we focus on a teacher's comments about the walkthrough as a useful curriculum planning resource. These comments were made during the debriefing interview that was 
conducted by the researchers after the classroom unit was completed. This was a teacher who had taught at the elementary level for over 25 years, who was confident in her abilities as a classroom teacher, and who described herself as someone who had used technologies over the past 20 years to support student learning. She reported that she had used games in the past, but had never planned a whole unit around a digital game before. For her, the walkthroughs meant that she "didn't have to play the game," she could "just read about what happened in a level" and then plan what her lessons around the content. For her, the walkthrough became a paratextual resource that allowed her to bypass gameplay skill and focus on "what mattered" - developing rich learning activities and assessments that linked to meaningful learning outcomes grounded in the events that unfolded in Sprite's Quest.

In the second example, a teacher recounted how she had a "high functioning, "A" female student who was particularly anxious about her own gameplay abilities. According to the teacher, the student outright refused to play the game. To ensure the student would be able to keep up with her peers, the walkthrough was made available so that she could complete the activities and assessments (as best she could) without really ever trying to play. In this case, the walkthrough became a kind of abstracted form of research (abstracted because the game was not played) and functioned instead as a literal text.

\section{Discussion}

In each of the three examples from our fieldwork, we describe the ways walkthroughs were introduced to in/formal learning environments, as well as the ways they were (or were not) taken up by their audiences. By describing where and how our participants did or did not take up the walkthroughs, our goal was to assist other researchers from having to 'reinvent the wheel'. We now turn our attention to the broader implications of our observations both for the use of walkthroughs as a scaffold for game-based pedagogy, but also some of the broader curriculum objectives that can be obtained by their inclusion in the middle school classroom.

\subsection{Lessons Learned}

We selected text-based walkthroughs [20, 36] for both the Wind Waker and the Lost Winds 2 studies because the overarching project is focused on the narrative aspects of games. The act of reading was assumed to be a better 'fit' for the project's goals yet proved to be a poor fit for our participants' preferred method for working through the puzzles of Wind Waker. Students sought out alternative paratexts, finding video walkthroughs to be more helpful to their play experience. This was in part because they found it difficult to recognize where they were in the game based on how the text described their location. Instead, it was much easier to match up the video on the iPad with their gameplay on the television screen to get back on track. Further, we hypothesize that the video walkthroughs were much less like school reading that typically involves working one's way through a very long, text-heavy document, and therefore, simply more appealing to those students who gravitated towards video walkthroughs.

This is not to say that text-based walkthroughs are something to be avoided - Apperley and Beavis [1] argued that translating written text into in-game actions is knowledge transformation in action. Instead, the uptake (or lack thereof) of walkthroughs in the lunchtime gaming club is further evidence that the surrounding environment will influence how games and paratexts are consumed. Examples of framing failing to explain to participants the utility of the walkthroughs include where they were completely avoided lest one be accused of being a cheat, and also where reading might have been too "school-like" for the lunchtime club participants. Our primary lesson learned from the Wind Waker study is to properly introduce walkthroughs and provide a demonstration to participants modeling how to navigate from text on the page to actions on the screen.

In the Lost Winds 2 study, where the walkthroughs had an explicit purpose and were consistently referred to by the teachers, researchers, and other students as a means of advancing in the game, they served as significant scaffolding both for skilled and less-skilled players. As one of the first students to complete the game noted: "the walkthrough made it SO much easier to complete the game quickly." And yet, despite these benefits, students seemed to 'forget' about the walkthroughs sitting on their desks as soon as the iPads were put away and they were asked to complete the written discussion questions asking them to recite and reflect on the elements of the narrative they encountered during the day's play session. Moving forward, we recognize that if we had spent additional time framing the Lost Winds 2 walkthrough as a resource not only for play, but also to serve as a suitable reference for the daily questions, students may not have approached the text with the tunnel vision we observed.

Finally, in the third study, teachers used the walkthrough written by the research staff as a peripheral text to inform them of the progression of the game without having to take the time to complete it 
themselves. Teachers also reported using the walkthroughs to better understand student progression through the game, and as a means of something to refer to when producing worksheets and other methods of evaluation. This was an unexpected outcome of use for the walkthroughs, as they were not a definitive 'text' on or about the game, and yet because they were in a recognizable 'school' format, they were given the authority to 'speak for' the game, as it were. The walkthroughs served further as an alternative learning text for those frustrated and/or unwilling to play the game, though that was a much less frequent occurrence. Interestingly, most students ignored the walkthroughs entirely, especially as the game was a side scrolling game with no puzzles to solve. When students were 'stuck' in the game it was usually because they had trouble with the controls or were simply trying to advance too quickly.

While we as researchers had assumed that the value of the different walkthroughs we implemented would be self-evident, the partial, and sometimes unwilling uptake of them by both student and teacher participants serves as a reminder that learning to play a game and being able to engage with its associated paratexts(s) is yet another form of information literacy. In the first study, our partner teacher was unfamiliar with walkthroughs and so she referred to them as "cheats". Despite being in an out of school setting (an optional, lunchtime club), having an authority figure refer to something as 'cheating' appeared to be enough to mark it as something that should be avoided. In the second study, the walkthroughs, while used by students, were sometimes difficult to 'decode'. That is, the students had a difficult time figuring out where they were in the game and how that related to where they were in a completely textual walkthrough (there were no images). Finally, in the third study, the walkthroughs became for the teachers a 'text' about the game, yet were largely ignored by the student participants.

This reluctance to engage in particular paratexts was surprising, as the literature that we reviewed earlier found that students were generally amenable to these texts in their classrooms. For example, Walsh and Apperley state, "...it became evident that digital game paratexts were familiar and significant to pupils. Through discussions with the teachers, we agreed the reading, writing, and designing of digital game paratexts would offer a tangible means by which to genuinely capitalize on pupil's out-of-school literacy practices, to intentionally valorize their gaming literacies and provide a platform to introduce digital games into the curriculum" [35:325]. This was only true for some of our participants, in particular, those who had prior access to videogames in their leisure spaces. For others, many of whom had little to no access to videogames, the walkthroughs became another 'literacy' that needed to be decoded, alongside or in addition to the game.

Interestingly, we observed in all three studies that students and teachers alike had difficulties moving between the documentation and descriptions in the walkthroughs and what was happening on screen. For example, they often could not identify the name of the level or the zone in which their avatar was standing, and therefore had difficulties recognizing what part of the walkthrough aligned specifically with their location in the game. Further, and especially for the novices, recognizing significant game-based events was problematic, meaning that they had no 'anchor' with which to identify the signposts that were outlined in the walkthroughs. Walsh and Apperley, were working with an older demographic of high school students, did not report these difficulties. This suggests the need for further investigations about what happens in the intervening years between Grade 6 and high school to better understand how students are introduced to and then eventually come to see these paratexts as familiar and significant.

\subsection{Curriculum Objectives}

Like Vie [34], we agree that walkthroughs share similarities with other technical documentation, including technical software texts. The ability to follow along in a text and then apply that information to what is going on in the screen is a significant information literacy piece that is currently missing from school curricula. In fact, a leading media literacy organization in Canada, "Media Smarts", has no reference to walkthroughs or even technical documentation like software manuals as an important $21^{\text {st }}$ century skill. Such cross-reading competencies are consistently absent from regional reports and investigations into transformative learning models for the $21^{\text {st }}$ century despite frequent references to the need for "emphasizing an interdisciplinary approach to learning" in the light of ubiquitous digital technologies" [4:4]. Additionally, research cited in Ministry documents exhibits the same enthusiasm for technology-enabled learning as first research forays into this area [25, 27] situating "increased student engagement" and tightened "problem solving skills" [23], as hopeful narratives that learning supported by digital technologies can increase communication, critical thinking and collaborative competencies relevant to a modern global landscape.

Media literacy curriculum prioritizes "interconnected skills". This asks that students be able to understand how a combination of "listening, 
speaking, reading and writing help them make sense of ... media texts" [22:117]. In all instances, walkthroughs were employed to facilitate such crossdisciplinary skills by allowing participants to self-solve software problems, essentially reading a walkthrough like a manual helping them troubleshoot and follow along with their own progress.

\section{Conclusion}

By taking a critical eye to revisit the use of walkthroughs in three separate scenarios, we are reminded of the importance of meeting students where they are. While Walsh and Apperley [35] were able to tap into the pre-existing gaming literacies of their participants, the students in our own studies are far from being "digital natives". Despite showing an enthusiasm for games in a lunchtime club or in their classroom, not students all have hands-on experience playing a game or navigating a paratext such as a walkthrough. Rather than assuming that middle school students have the gaming literacy to understand how and where to apply the knowledge from a walkthrough to their in-game activities, our findings suggest that scaffolding will be helpful in future implementations of walkthroughs in in/formal learning environments. By providing detailed explanations of what walkthroughs are, how to read them, and how to translate their information to one's videogame play, we can ensure that all students start with equal footing. By sharing our experiences using walkthroughs in the field - both what worked and what failed - in this paper we have sought to demonstrate that while walkthroughs can further digital media related curriculum goals, their use should be treated like any other form of literacy, that is, one that must be properly scaffolded throughout the curriculum.

Walkthroughs in each of the educational contexts described in this paper are, whether taken as paratexts or as literal texts for learning, a potentially understudied resource for supporting game-based learning in the classroom. In particular, they have the possibility to help level the playing field, giving novices access to the affordances, puzzles and other "hidden" characteristics of games in ways that are already available to more expert players. And yet, for some participants, walkthroughs remained a text that they were not able to decode in relation to their progress in the game, providing further evidence that not all students enter the classroom as digital natives, and therefore particular knowledge(s) should not be assumed. Given the value of walkthroughs as significant forms of technical documentation, and the importance of cross-reading as a $21^{\text {st }}$ century skill, this research serves to support the argument that walkthroughs can be used as a central component of media literacy curricula. We also hoped to show how walkthroughs do serve as a valuable resource for some, particularly those less familiar with digital games, supporting them to advance in a game, or at least to 'read' the game as a text that has a resonance with other forms of reading and writing in school contexts.

\section{References}

[1] Apperley, T., and C. Beavis, "Literacy into action: digital games as action and text in the English and literacy classroom", Pedagogies: An International Journal 6(2), 2011, pp. 130-143.

[2] Bergstrom, K., S. de Castell, and J. Jenson, "Digital Detritus: What Can We Learn From Abandoned Massively Multiplayer Online Game Avatars?", Proceedings of the First International Joint Conference of DiGRA and FDG, (2016).

[3] Bergstrom, K., J. Jenson, R. Hydomako, and S. de Castell, "The keys to success: Supplemental measures of player expertise in Massively Multiplayer Online Games", Journal of Gaming \& Virtual Worlds 7(1), 2015, pp. 101121.

[4] Boudreault, F.-A., J. Haga, P. Paylor, A. Sabourin, S. Thomas, and C. van der Linden, Future Tense: Adapting Canadian Education Systems for the 21st Century, Action Canada, Ontario, 2013.

[5] Braithwaite, A., “'Seriously, get out': Feminists on the forums and the War(craft) on women", New Media \& Society 16(5), 2014, pp. 703-718.

[6] Brock, A., "“When Keeping it Real Goes Wrong': Resident Evil 5, Racial Representation, and Gamers", Games and Culture 6(5), 2011, pp. 429-452.

[7] Burn, A., "Writing computer games: game literacy and new-old narratives", L1 Educational Studies in Language and Literature 7(4), 2007, pp. 45-67.

[8] Carter, M., "Emitexts and Paratexts: Propaganda in EVE Online", Games and Culture 10(4), 2015, pp. 311-342.

[9] de Castell, S., E. Flynn-Jones, J. Jenson, and K. Bergstrom, "Learning Links: A study of narrative learning through games with The Legend of Zelda:Windwaker", Proceedings of the 50th Hawaii International Conference on System Sciences, (2017), 2016-2025.

[10] Chen, M., Leet Noobs: The Life and Death of an Expert Player Group in World of Warcraft, Peter Lang, New York, 2012. 
[11] Consalvo, M., "Zelda 64 and Video Game Fans: A Walkthrough of Games, Intertextuality, and Narrative", Television \& New Media 4(3), 2003, pp. 321-334.

[12] Consalvo, M., Cheating: Gaining Advantage in Videogames, MIT Press, Boston, MA, 2007.

[13] Fisher, S., J. Jenson, and S. de Castell, "Dynamic (con)texts: Close readings of girls' video game play", In C. Bradford and M. Reimer, eds., Girls, Texts, Cultures. Wilfred Laurier Press, Waterloo, ON, 2015, 261-286.

[14] Gee, J.P., What video games have to teach us about learning and literacy, Palgrave Macmillan, New York, 2007.

[15] Giddings, S., "Playing with Non-Humans: Digital Games as Technocultural Form", In S. De Castell and J. Jenson, eds., Worlds in play: international perspectives on digital games research. Peter Lang, New York, 2007, 115128.

[16] Jenkins, H., “Quentin Tarantino's Star Wars? Digital Cinema, Media Convergence, and Participatory Culture", In M.G. Durham and D. Kellner, eds., Media and cultural studies: Keyworks. Blackwell, Malden, MA, 2006, 549-576.

[17] Jenkins, H., ed., Confronting the challenges of participatory culture: Media education for the 21st Century, MIT Press, MA, 2009.

[18] Juul, J., "The Open and the Closed: Games of Emergence and Games of Progression", Digital Cultures Conference Proceedings, Tampere University Press (2002), 323-329.

[19] Kringiel, D., "Learning to Play: Video Game Literacy in the Classroom", In J. Fromme and A. Unger, eds., Computer Games and New Media Cultures. Springer Netherlands, Dordrecht, 2012, 633-646.

[20] kymtendo, "LostWinds: Winter of the Melodias", GameFAQs, 2009. https://www.gamefaqs.com/wii/971949lostwinds-winter-of-the-melodias/faqs/58164

[21] Malone, K.-L., "Dragon Kill Points: The Economics of Power Gamers", Games and Culture 4(3), 2009, pp. 296316.

[22] Ministry of Education, The Ontario Curriculum Grades 1-8: Language, 2006.

[23] Ontario Public Service, 21st Century Competencies: Towards Defining 21st Century Competencies for Ontario, 2016.

[24] Paul, C.A., "Welfare Epics? The Rhetoric of Rewards in World of Warcraft", Games and Culture 5(2), 2010, pp. 158176.
[25] Prensky, M., "Digital Natives, Digital Immigrants", On the Horizon 9(5), 2001.

[26] Prensky, M., Don't bother me mom - I'm learning!: How computer and video games are preparing your kids for 21 st century success and how you can help!, Paragon House., St. Paul, MN:, 2006.

[27] Shaffer, D.W., K.R. Squire, R. Halverson, and J.P. Gee, "Video games and the future of learning", Phi Delta Kappan 87(2), 2005, pp. 104-111.

[28] Stanley, G., and K. Mawer, "Language Learners \& Computer Games: From Space Invaders to Second Life", Teaching English as a Second or Foreign Language 11(4), 2008, pp. 1-12.

[29] Steinkuehler, C., E. Alagoz, E. King, and C. Martin, “A Cross Case Analysis of Two Out-of-School Programs Based on Virtual Worlds", International Journal of Gaming and Computer-Mediated Simulations 4(1), 2012, pp. 25-54.

[30] Steinkuehler, C., and E. King, "Digital literacies for the disengaged: creating after school contexts to support boys' game-based literacy skills", On the Horizon 17(1), 2009, pp. 47-59.

[31] Steinkuehler, C., E. King, E. Alagoz, et al., "Let Me Know When She Stops Talking: Using Games for Learning Without Colonizing Play", Proceedings of the 7th international conference on Games + Learning + Society Conference, ETC Press (2011), 210-220.

[32] Taylor, T.L., Play Between Worlds: Exploring Online Game Culture, The MIT Press, 2006.

[33] Van Eck, R., "Digital Game-Based Learning: It's Not Just the Digital Natives Who Are Restless", 2006.

[34] Vie, S., "Tech Writing, Meet Tomb Raider: video and computer games in the technical communication classroom", E-Learning 5(2), 2008, pp. 157.

[35] Walsh, C., and T. Apperley, "Using gaming paratexts in the literacy classroom", Games + Learning + Society Conference, (2012), 322-329.

[36] "The Game Begins", Thonky.com, 2015. http://www.thonky.com/zelda-wind-waker/the-game-begins 